\title{
BMJ Open Safety and feasibility of transcranial direct current stimulation (tDCS) combined with sensorimotor retraining in chronic low back pain: a protocol for a pilot randomised controlled trial
}

\author{
Adam Louis Ouellette, ${ }^{1}$ Matthew B Liston, ${ }^{1}$ Wei-Ju Chang, ${ }^{1}$ David M Walton, ${ }^{2}$ \\ Benedict Martin Wand, ${ }^{3}$ Siobhan M Schabrun ${ }^{1}$
}

To cite: Ouellette AL, Liston MB, Chang W-J, et al. Safety and feasibility of transcranial direct current stimulation (tDCS) combined with sensorimotor retraining in chronic low back pain: a protocol for a pilot randomised controlled trial. BMJ Open 2017;7:e013080. doi:10.1136/ bmjopen-2016-013080

- Prepublication history and additional material is available. To view please visit the journal (http://dx.doi.org/ 10.1136/ bmjopen-2016-013080).

Received 23 November 2016 Revised 30 January 2017 Accepted 23 February 2017

\section{CrossMark}

${ }^{1}$ Brain Rehabilitation and Neuroplasticity Unit, School of Science and Health, Western Sydney University, Penrith, New South Wales, Australia

${ }^{2}$ School of Physiotherapy, Western University, Elborn College, London, Canada

${ }^{3}$ The University of Notre Dame Australia, The University of Western Sydney, Penrith, New South Wales, Australia

Correspondence to Dr Siobhan M Schabrun; s.schabrun@uws.edu.au

\section{ABSTRACT}

Introduction Chronic low back pain (LBP) is a common and costly health problem yet current treatments demonstrate at best, small effects. The concurrent application of treatments with synergistic clinical and mechanistic effects may improve outcomes in chronic LBP. This pilot trial aims to (1) determine the feasibility, safety and perceived patient response to a combined transcranial direct current stimulation (tDCS) and sensorimotor retraining intervention in chronic LBP and (2) provide data to support a sample size calculation for a fully powered trial should trends of effectiveness be present.

Methods and analysis A pilot randomised, assessor and participant-blind, sham-controlled trial will be conducted. Eighty participants with chronic LBP will be randomly allocated to receive either (1) active tDCS + sensorimotor retraining or (2) sham tDCS + sensorimotor retraining. tDCS (active or sham) will be applied to the primary motor cortex for 20 min immediately prior to 60 min of supervised sensorimotor retraining twice per week for 10 weeks. Participants in both groups will complete home exercises three times per week. Feasibility, safety, pain, disability and pain system function will be assessed immediately before and after the 10-week intervention. Analysis of feasibility and safety will be performed using descriptive statistics. Statistical analyses will be conducted based on intentionto-treat and per protocol and will be used to determine trends for effectiveness.

Ethics and dissemination Ethical approval has been gained from the institutional human research ethics committee (H10184). Written informed consent will be provided by all participants. Results from this pilot study will be submitted for publication in peer-reviewed journals. Trial registration number ACTRN12616000624482

\section{INTRODUCTION}

Low back pain (LBP) is one of the most common reasons for people to seek healthcare. ${ }^{1}$ Of those that report LBP, 44\%-78\% experience a re-occurrence within 12 months, ${ }^{2}$ and approximately $25 \%$ of individuals develop chronic pain. ${ }^{3}$ Despite high

\section{Strengths and limitations of this study}

- A randomised, assessor and participant-blind, sham-controlled trial that will provide the first information on the feasibility and safety of a combined transcranial direct current stimulation and sensorimotor retraining intervention in chronic low back pain.

- This pilot trial is not powered to determine treatment effectiveness. However, if trends of effectiveness are present, these data will support a fully powered trial in future.

- The treating physiotherapist is not blinded to group allocation.

prevalence, current treatments for chronic LBP demonstrate, at best, small effect sizes. ${ }^{45}$ One avenue to improve outcomes in chronic LBP is through the application of combined treatments with synergistic clinical and mechanistic effects.

Sensorimotor retraining is a novel treatment that incorporates motor control exercise and lumbar tactile retraining and has been shown to be effective in early randomised controlled trials and case studies of chronic LBP. ${ }^{6-9}$ The mechanism underpinning improvements in pain and function with sensorimotor retraining is thought to involve normalisation of motor and sensory cortical changes and improved pain system function. ${ }^{69}$ The addition of a second treatment approach that targets synergistic mechanisms may boost the effectiveness of sensorimotor retraining in people with chronic LBP.

Transcranial direct current stimulation (tDCS), a form of non-invasive brain stimulation, is thought to promote cortical plasticity $^{10-12}$ and improve pain system function through direct effects on the cortex and 
thalamus, ${ }^{13-19}$ as well as 'downstream' effects on the anterior cingulate cortex and upper brainstem. ${ }^{20}{ }^{21}$ Studies of healthy individuals and people with some forms of chronic pain suggest that anodal tDCS applied to the primary motor cortex can reduce pain. ${ }^{18} 22-24$ Indeed, a recent systematic review in fibromyalgia demonstrates the effects on pain that are analogous to those of FDA-approved pharmaceuticals with considerably fewer side effects. ${ }^{25}$ In addition, the cortical effects of tDCS are hypothesised to increase the brain's receptiveness to other treatments, a phenomenon known as priming. ${ }^{26}{ }^{27}$ Based on these mechanisms, tDCS may optimise the responsiveness of the brain to sensorimotor retraining as well as target synergistic mechanisms of sensorimotor cortex reorganisation promoted by sensorimotor retraining. The complementary mechanistic targets of these treatments may summate to improve clinical outcomes beyond that which can be achieved with sensorimotor retraining alone. Despite this, no study has examined the effect of a combined tDCS and sensorimotor retraining therapy in chronic pain. ${ }^{28-30}$

This pilot randomised controlled trial (RCT) protocol will outline study methods and resources required to determine the feasibility, acceptability and safety ${ }^{31}$ of tDCS combined with sensorimotor retraining for people with chronic LBP. This protocol uses a pain and neuroscience mechanisms approach to combine two treatments with the potential to produce complementary and additive effects on sensorimotor cortical organisation and pain system function. The specific aims are to (1) determine the feasibility, safety, perceived patient response to, and acceptability of, a combined tDCS and sensorimotor training intervention in chronic LBP and (2) provide data to support a sample size calculation for a fully powered trial should trends of effectiveness be present.

\section{METHODS AND ANALYSIS}

Trial design

We will use a pilot randomised, participant and assessor-blind controlled trial design. The trial will be conducted and reported according to the Consolidated Standards of Reporting Trials (CONSORT) statement for non-pharmacological treatment standards and the Template for Intervention Description and Replication checklist and guide. ${ }^{32}$ The trial has been prospectively registered with the Australian and New Zealand Clinical Trials Registry (ACTRN12616000624482).

\section{Participants}

Participants aged between 18 and 60 years with chronic LBP will be recruited from the Western Sydney suburbs, in New South Wales, Australia. Chronic LBP is defined as pain occurring between the bottom rib and the gluteal fold, which has been present for more than 12 weeks. ${ }^{243}$ Participants will be required to have an average pain score greater than or equal to $4 / 10$ on a numerical rating scale in the week prior to enrolment ${ }^{34}$ and a minimum score of 4 points on the Roland Morris Disability Questionnaire
(RMDQ) to limit the potential for floor effects. ${ }^{6}$ Participants will be excluded if they (1) present with specific spinal pathology (tumour, spondylolythesis, fracture, etc), nerve root pain or co-existing major muscular, joint, neurological or psychiatric conditions; (2) have undergone back surgery; (3) are currently undertaking a structured exercise programme for LBP; or (4) present with contraindications to tDCS (eg, cuts or blisters under the electrode sites) or conditioned pain modulation techniques (eg, loss of sensation). Participants can continue to use their normal medication for the duration of the trial. The type of medication and dosage used will be recorded at the baseline assessment.

\section{Recruitment}

Participants will be recruited from local healthcare providers (eg, medical practitioners, chiropractors and physiotherapists), support groups, social media and newspaper advertisements. Potential participants will first complete an online screening questionnaire with those who meet the inclusion criteria contacted by the investigators to arrange baseline assessment. Participants will then provide written informed consent on arrival at the baseline assessment. The number of people screened and enrolled in the trial, as well as reasons for ineligibility, will be recorded.

\section{Randomisation}

Participants will be individually randomised on a 1:1 basis to the active or control groups in equal numbers. The randomisation schedule will be concealed in consecutively numbered, sealed opaque envelopes. An investigator not involved in recruitment, treatment or assessment will provide the envelope to the treating clinician who will reveal group allocation.

\section{Blinding}

Participants, the therapist and the outcome assessor will be blind to group allocation. The tDCS unit used to deliver the direct current stimulation includes a blinded study mode that allows the therapist to enter only a blinded randomisation code to determine whether active or sham stimulation is delivered. Set-up of the randomisation code, and programming of the tDCS unit, will be performed by an investigator not involved in the trial. The success of participant blinding will be assessed at follow-up assessment using a yes/no response to the question, 'Do you feel you received the real brain stimulation?' and a $10 \mathrm{~cm}$ visual analogue scale of the individual's confidence in that judgement. ${ }^{35} 36$ Participants will also be asked, 'Why do you believe you received the real/sham brain stimulation?' and, 'Was it divulged to you whether you were receiving real brain stimulation or not?' The success of therapist and assessor blinding will be determined at the completion of the follow-up assessment for each participant using a yes/no response to the question, 'Did you know the intervention group to 
which the participant was assigned before trial completion?' and, 'If you answer "yes", how was it divulged to you?'. 35

\section{Intervention}

Participants will be randomly allocated to receive either (1) active tDCS + sensorimotor retraining or (2) sham tDCS + sensorimotor retraining. The intervention will be delivered twice a week for 10 weeks and will consist of 20 min of active or sham tDCS immediately followed by 1 hour of supervised sensorimotor retraining. A 10-week intervention has been chosen as this duration has led to improved outcomes in people with chronic LBP following a sensorimotor retraining intervention in a previous study. ${ }^{6}$ A qualified physiotherapist, trained in the use of tDCS, will deliver both the tDCS intervention and the sensorimotor retraining in a consulting room of Western Sydney University. To replicate typical clinical practice, participants in both groups will also complete home exercise three times per week. Outcome measures will be assessed immediately before and immediately after the 10 -week intervention.

\section{Transcranial direct current stimulation}

tDCS will be delivered to the primary motor cortex using a DC-STIMULATOR PLUS, (NeuroConn, Ilmenau, Germany), while participants are comfortably and quietly seated. Direct current will be delivered for $20 \mathrm{~min}$ via two $35 \mathrm{~cm}^{2}$ surface sponge electrodes. The active electrode (anode) will be positioned over the primary motor cortex using the International 10-20 system contralateral to the side of worst LBP. The reference electrode (cathode) will be positioned over the contralateral supraorbital region ipsilateral to the side of pain. The primary motor cortex has emerged as one of the most effective and reliable sites for tDCS in the treatment of pain, producing improvements in pain analogous to those of FDA-approved pharmaceuticals in other musculoskeletal pain conditions with considerably fewer side effects. ${ }^{25}$ Using standard tDCS parameters, ${ }^{25}$ current intensity will be ramped up (0-1 mA) and down (1-0 mA) over $10 \mathrm{~s}$ at the beginning and end of the 20 min stimulation period. For sham stimulation, electrodes will be placed in an identical position. To provide the initial itching sensation, stimulation will be turned on for $15 \mathrm{~s}$ and then off. Participants will be informed that they may or may not perceive any sensation during the treatment. This procedure has been shown to effectively blind participants to the stimulation condition at intensities of $1 \mathrm{~mA} .^{37}$

\section{Sensorimotor retraining}

Immediately following the active or sham tDCS intervention, participants will commence a graded sensorimotor retraining programme informed by a previously published protocol. ${ }^{67}$ Components of the protocol include progressive tactile localisation, tactile discrimination and graphaesthesia training, laterality recognition, imagined movements, precision focused and feedback enriched movement training (including full range movements, isometric local muscle recruitment and co-contraction and dissociation exercises) and precision focused and feedback enriched functional retraining. ${ }^{6}$ Five stages exist for each of the sensory and motor retraining elements. Participants will be progressed through each stage by the physiotherapist based on specific, previously published criteria. $^{6}$

Participants will be provided with a home exercise diary containing visual and written instructions for each exercise (including dosage) and will be asked to practice the training at home for $30 \mathrm{~min}, 3$ times per week. The exercise diary will include space for participants to outline which exercises were completed, how many repetitions were performed and any comments regarding the home exercise programme (eg, whether pain was present, whether any exercises were difficult and if applicable, the reason why exercises were unable to be completed). The exercise diary will be returned to the investigator at the postintervention assessment session.

\section{OUTCOME MEASURES}

Measures of feasibility, safety and adherence will be collected throughout the pilot study, while endpoint measures of pain and function (Brief Pain Inventory, RMDQ) as well as pain mechanisms will be measured 1 week prior to the participant commencing, and within 1 week of completion, of the 10-week intervention. All measures will be performed in the research laboratories of Western Sydney University.

\section{Primary outcomes}

Feasibility

The number of participants that (1) meet the inclusion criteria, (2) agree to be randomised, (3) complete the intervention and (4) attend the postintervention assessment will be calculated in accordance with CONSORT guidelines. Feasibility will be measured as (1) the number of treatment sessions attended by each participant, (2) number of drop-outs in each group, (3) proportion of participants recruited from the total number screened, (4) willingness of each participant to undergo therapy on an 11-point numerical rating scale with 'not at all willing' at 0 and 'very willing' at 10 (measured at baseline) and (5) the number of home exercise sessions completed.

\section{Safety}

Safety will be assessed as any adverse effect, defined as 'a response to an intervention which is noxious and unintended, and which occurs at doses normally used in man for the prophylaxis, diagnosis, or therapy of disease, or for the modification of physiological function' and that likely has a causal relationship with the intervention, ${ }^{38}$ reported on verbal questioning by the treating physiotherapist at each session. A mild tingling or itching sensation under the electrodes, fatigue, headache, nausea and insomnia have been reported as potential adverse reactions 
following tDCS. ${ }^{39}$ Potential adverse reactions as a result of sensorimotor retraining may include increased pain or muscle soreness in the back. The treating physiotherapist will record a description of any adverse effects along with the severity and duration of symptoms and how the adverse effect was managed.

\section{Secondary outcomes \\ Questionnaires}

The Brief Pain Inventory will be used to measure pain severity and disability. To assess pain, participants will be asked to complete four numerical rating scales anchored with 0 ('no pain') and 10 ('worst pain imaginable') for pain at its (1) most intense over the last week, (2) least intense over the last week, (3) average intensity over the last week and (4) right now. Scores from each scale will be averaged to calculate a final pain severity score out of $10{ }^{40}$ To assess function, participants will complete seven numerical rating scales to describe how their back pain interfered with daily life (eg, general activity and mood) in the past week. Each scale will be anchored with 0 ('does not interfere with daily life') and 10 ('completely interferes'). Scores from the seven scales will be averaged to give a final pain interference score out of $10{ }^{41}$ The Brief Pain Inventory has been shown to be valid and reliable in the chronic LBP population. ${ }^{40}$ Self-reported disability will also be measured using the 24-point RMDQ that has been shown to be valid and reliable in people with LBP. $^{42}{ }^{43}$ Finally, the global perceived effect of treatment scale, where each participant's perceived response to therapy is assessed using an 11-point Likert scale ranging from 'vastly worsened' to 'completely recovered', will be completed. ${ }^{44}$

\section{Measures of pain mechanisms}

Measures of pain mechanisms will be performed in the same order for all participants.

\section{Secondary outcomes of pain mechanisms \\ Pressure pain thresholds (PPTs)}

Pressure pain thresholds (PPTs) will be measured using a hand-held pressure algometer (Algometer Type II, SBMEDIC Electronics, Sweden) with a probe size of $1 \mathrm{~cm}^{2}$. The probe will be applied perpendicular to the skin (rate $40 \mathrm{kPa} / \mathrm{s}$ ) until the participant first reports that the sensation of pressure has changed to pain. PPTs will be measured three times, in random order, at each of nine sites on a $3 \times 3$ grid (spacing $2 \mathrm{~cm}$ between points, 27 stimuli in total) centred at the site of worst pain. Participants will be asked to locate the site of worst pain at baseline for positioning of the grid. The location of this site will be recorded using bony landmarks to ensure that the same site is targeted in the follow-up assessment. In addition, three PPTs will be measured at the contralateral thumbnail. The average of the three measurements at each site will be used for analyses. PPT measures have been shown to be reliable in chronic LBP. $^{45} 46$

\section{Heat pain thresholds}

Heat pain thresholds (HPT) will be measured using a Thermal Sensory Analyzer system (TSA-2001, Q-Sens-CPM, Medoc, Ramat Yishai, Israel). A $30 \times 30 \mathrm{~mm}$ Peltier-based thermode will be placed on the skin. The temperature will start at $32^{\circ} \mathrm{C}$ and increase at a rate of $0.5^{\circ} \mathrm{C} / \mathrm{s}$. Participants will push a button when the sensation of heat first turns to a sensation of pain. HPTs will be measured at (1) the site of worst pain, (2) the lumbar site contralateral to the side of pain and (3) the ventral aspect of the forearm contralateral to the side of pain $(10 \mathrm{~cm}$ distal from the elbow crest). Three measurements will be recorded at each site and the average analysed. HPT measures are reliable in chronic LBP. ${ }^{45}$

\section{Conditioned pain modulation}

Conditioned pain modulation (CPM) is examined as a change in the pain perceived in one body region (test stimulation) as a result of pain induced in another body region (conditioned stimulation). It is a safe measure of pain processing that is thought to indicate the function of descending pain control systems. We will use pressure pain (PPTs) as the test stimulation and heat pain $\left(1^{\circ} \mathrm{C}\right.$ above HPT) as the conditioned stimulation (Thermal Sensory Analyzer, TSA-2001, Q-Sense-CPM, Medoc, Ramat Yishai, Israel). Three PPTs will be measured before the application of heat pain. Heat pain will then be applied via a $30 \times 30 \mathrm{~mm}$ thermode with three sequential PPT measures taken after $30 \mathrm{~s}$ of the conditioning (heat) stimulus. The heat stimulus will then be removed. Participants will be asked to rate their pain during conditioning (heat) stimulation on a numeric rating scale $(0-100)$ at 0 s, 30 s and at the end of the trial. Pain scores will be maintained between 50 and 80/100 for the conditioned stimulus during testing. Participants will complete two trials in random order: (1) test stimulation applied at the most painful lumbar region (indicated by the participant) and conditioned stimulation at the contralateral forearm and (2) test stimulation at the forearm ipsilateral to the site of pain and conditioned stimulation at the contralateral lumbar region. The CPM paradigm is reliable in chronic LBP. $^{45} 4849$

\section{Temporal summation}

Temporal summation (TS) will be assessed using a $26 \mathrm{~g}$ nylon monofilament (Aesthesio: DanMic Global) to apply repeated mechanical stimulation according to the Standardized Evaluation of Pain protocol. ${ }^{50}$ The participant will be asked whether a single filament stimulus provokes pain. If the answer is 'yes', the participant will then be asked to rate the pain on a numeric rating scale $(0-100)$. If the answer is 'no', a 'zero' will be recorded on a numeric rating scale. The filament will then be applied to the skin at a rate of $1 \mathrm{~Hz}$ for $30 \mathrm{~s}$. The participant will be asked to rate the pain on the numeric rating scale again at the end of the 30 stimuli. TS will be tested on the most painful area and the dorsal aspect of the non-dominant wrist joint. Previous work has recommended using TS of 
mechanical pain to assess endogenous pain modulation in chronic LBP populations. ${ }^{51}$ TS of mechanical pain is a reliable test. ${ }^{52}$

\section{Data and statistical analyses}

Data for feasibility and safety will be analysed using descriptive statistics. Trends for effectiveness will be determined in two ways: (1) to assess within group changes in pain, function and pain mechanisms, a one-way repeated measures analysis of variance will be performed to compare baseline and 10-week follow-up scores for each outcome, in each group; (2) to assess betweengroup changes in pain, function and pain mechanisms, an analysis of covariance will be performed where group allocation is the fixed factor and the corresponding baseline outcome values are included as covariates. ${ }^{53}$ Post hoc Bonferroni tests will be applied where appropriate. Effect sizes will be determined using partial $\eta^{2}$ from planned contrasts. Alpha will be set at 0.05 . As this is a pilot trial, missing data will not be replaced.

The size of the treatment effects will be used to determine whether a full randomised controlled trial is warranted. ${ }^{54}$ Means and SD for measures of pain, disability and pain mechanisms will be used to perform a sample size estimate. Power will be set at $80 \%$ to detect between-group differences with an alpha of 0.05 and a drop-out rate based on that of the pilot trial. SigmaPlot software will be used to analyse all data.

\section{Sample size}

This is a pilot study that will be used to generate data to inform a future full-scale randomised controlled trial should the intervention appear feasible, safe and show trends of effectiveness. As a result, a prospective sample size calculation was not conducted. Based on projected recruitment rates within the study timeframe, a sample size of 40 participants per group (80 in total) has been selected.

\section{ETHICS AND DISSEMINATION}

Western Sydney University Human Research Ethics Committee has approved this trial (H10184). All participants will provide written informed consent following verbal and written explanation of the study protocol and the opportunity to ask questions. Results will be presented at scientific meetings and published in peer-reviewed journals. All publications and presentations related to the study will be authorised and reviewed by the study investigators.

\section{TRIAL STATUS}

This trial will begin recruitment in September 2017 and is expected to be complete (including follow-up testing) by December 2018 .
Contributors ALO, MBL, W-JC, DW, BMW and SMS were each involved in the conception, design, writing and editing of the study protocol. ALO, MBL, WJC, DW, BMW and SMS approved the final protocol.

Funding SMS holds a Career Development Fellowship (1105040) from the National Health and Medical Research Council of Australia (NHMRC). MBL receives salary support from the NHMRC (1059116). W-JC is the recipient of a postgraduate scholarship from the NHMRC (1094434).

Competing interests None declared.

Ethics approval Western Sydney University (H10184).

Provenance and peer review Not commissioned; externally peer reviewed.

Open Access This is an Open Access article distributed in accordance with the Creative Commons Attribution Non Commercial (CC BY-NC 4.0) license, which permits others to distribute, remix, adapt, build upon this work non-commercially, and license their derivative works on different terms, provided the original work is properly cited and the use is non-commercial. See: http://creativecommons.org/ licenses/by-nc/4.0/

(C) Article author(s) (or their employer(s) unless otherwise stated in the text of the article) 2017. All rights reserved. No commercial use is permitted unless otherwise expressly granted.

\section{REFERENCES}

1. Manek NJ, MacGregor AJ. Epidemiology of back disorders: prevalence, risk factors, and prognosis. Curr Opin Rheumatol 2005;4:324-30.

2. Airaksinen O, Brox JI, Cedraschi C, et al. Chapter 4. european guidelines for the management of chronic nonspecific low back pain. Eur Spine J 2006;15(Suppl 2):s192-s300.

3. Manchikanti L, Singh V, Datta S, et al. Comprehensive review of epidemiology, scope, and impact of spinal pain. Pain Physician 2009;12:E35-70.

4 Saragiotto BT, Maher CG, Yamato TP, et al. Motor control exercise for chronic non-specific low-back pain. Cochrane Database Syst Rev 2016;1:CD012004.

5. Saragiotto BT, Maher CG, Yamato TP, et al. Motor Control Exercise for nonspecific low back pain: a Cochrane Review. Spine 2016;41:1284-95.

6. Wand BM, O'Connell NE, Di Pietro F, et al. Managing chronic nonspecific low back pain with a sensorimotor retraining approach: exploratory multiple-baseline study of 3 participants. Phys Ther 2011;91:535-46.

7. Moseley GL, Zalucki NM, Wiech K. Tactile discrimination, but not tactile stimulation alone, reduces chronic limb pain. Pain 2008;137:600-8.

8. Wälti P, Kool J, Luomajoki H. Short-term effect on pain and function of neurophysiological education and sensorimotor retraining compared to usual physiotherapy in patients with chronic or recurrent non-specific low back pain, a pilot randomized controlled trial. BMC Musculoskelet Disord 2015;16:83.

9. Flor $\mathrm{H}$, Diers $\mathrm{M}$. Sensorimotor training and cortical reorganization. NeuroRehabilitation 2009;25:19-27.

10. Frazer A, Williams J, Spittles M, et al. Anodal transcranial direct current stimulation of the motor cortex increases cortical voluntary activation and neural plasticity. Muscle Nerve 2016;54:903-13.

11. Rroji O, van Kuyck K, Nuttin B, et al. Anodal tDCS over the Primary Motor Cortex facilitates Long-Term memory formation reflecting UseDependent plasticity. PLoS One 2015;10:e0127270.

12. Wessel MJ, Zimerman M, Hummel FC. Non-invasive brain stimulation: an interventional tool for enhancing behavioral training after stroke. Front Hum Neurosci 2015;9:265.

13. Boggio PS, Amancio EJ, Correa CF, et al. Transcranial DC stimulation coupled with TENS for the treatment of chronic pain: a preliminary study. Clin J Pain 2009;25:691-5.

14. Fregni F, Gimenes R, Valle AC, et al. A randomized, shamcontrolled, proof of principle study of transcranial direct current stimulation for the treatment of pain in Fibromyalgia. Arthritis Rheum 2006;54:3988-98.

15. Hummel F, Celnik P, Giraux P, et al. Effects of non-invasive cortical stimulation on skilled motor function in chronic stroke. Brain 2005;128:490-9.

16. Nitsche MA, Seeber A, Frommann K, et al. Modulating parameters of excitability during and after transcranial direct current stimulation of the human motor cortex. J Physiol 2005;568:291-303. 
17. Stagg CJ, Jayaram G, Pastor D, et al. Polarity and timing-dependent effects of transcranial direct current stimulation in explicit motor learning. Neuropsychologia 2011;49:800-4.

18. Zaghi S, Thiele B, Pimentel D, et al. Assessment and treatment of pain with non-invasive cortical stimulation. Restor Neurol Neurosci 2011;29:439-51.

19. Vaseghi B, Zoghi M, Jaberzadeh S. Does anodal transcranial direct current stimulation modulate sensory perception and pain? A metaanalysis study. Clin Neurophysiol 2014;125:1847-58.

20. García-Larrea L, Peyron R, Mertens P, et al. Electrical stimulation of motor cortex for pain control: a combined PET-scan and electrophysiological study. Pain 1999;83:259-73.

21. Strafella AP, Vanderwerf $Y$, Sadikot AF. Transcranial magnetic stimulation of the human motor cortex influences the neuronal activity of subthalamic nucleus. Eur J Neurosci 2004;20:2245-9.

22. O'Connell NE, Cossar J, Marston L, et al. Transcranial direct current stimulation of the motor cortex in the treatment of chronic nonspecific low back pain: a randomized, double-blind exploratory study. Clin J Pain 2013;29:26-34.

23. O'Connell NE, Wand BM. Transcranial direct current brain stimulation for chronic pain. BMJ 2015;350:h1774.

24. Schabrun SM, Jones E, Elgueta Cancino EL, et al. Targeting chronic recurrent low back pain from the top-down and the bottom-up: a combined transcranial direct current stimulation and peripheral electrical stimulation intervention. Brain Stimul 2014;7:451-9.

25. Marlow NM, Bonilha HS, Short EB. Efficacy of transcranial direct current stimulation and repetitive transcranial magnetic stimulation for treating Fibromyalgia syndrome: a systematic review. Pain Pract 2013:13:131-45.

26. Schabrun SM, Chipchase LS. Priming the brain to learn: the future of therapy? Man Ther 2012;17:184-6.

27. Stoykov ME, Madhavan S. Motor priming in neurorehabilitation. $J$ Neurol Phys Ther 2015;39:33-42.

28. Ji RR, Kohno T, Moore KA, et al. Central sensitization and LTP: do pain and memory share similar mechanisms? Trends Neurosci 2003;26:696-705.

29. Latremoliere A, Woolf CJ. Central sensitization: a generator of pain hypersensitivity by central neural plasticity. J Pain 2009;10:895-926.

30. Sharif-Naeini R, Basbaum Al. Targeting pain where it resides. In the brain. Sci Transl Med 2011;3:65ps1.

31. Plow EB, Malone DA, Machado A. Deep brain stimulation of the ventral striatum/anterior limb of the internal capsule in thalamic pain syndrome: study protocol for a pilot randomized controlled trial. Trials 2013;14:241.

32. Hoffmann TC, Glasziou PP, Boutron I, et al. Better reporting of interventions: template for intervention description and replication (TIDieR) checklist and guide. BMJ 2014;348:g1687.

33. van Tulder M, Becker A, Bekkering T, et al. Chapter 3. european guidelines for the management of acute nonspecific low back pain in primary care. Eur Spine J 2006;15(Suppl 2):s169-s191.

34. Lin WC, Yeh CH, Chien LC, et al. The Anti-Inflammatory actions of auricular point acupressure for chronic low back pain. Evid Based Complement Alternat Med 2015;2015:1-9.

35. Chang WJ, Bennell KL, Hodges PW, et al. Combined exercise and transcranial direct current stimulation intervention for knee osteoarthritis: protocol for a pilot randomised controlled trial. BMJ Open 2015;5:e008482.

36. O'Connell NE, Cossar J, Marston L, et al. Rethinking clinical trials of transcranial direct current stimulation: participant and Assessor blinding is inadequate at intensities of $2 \mathrm{ma}$. PLoS One 2012;7:e47514.
37. Gandiga PC, Hummel FC, Cohen LG. Transcranial DC stimulation (tDCS): a tool for double-blind sham-controlled clinical studies in brain stimulation. Clin Neurophysiol 2006;117:845-50.

38. Carlesso LC, Macdermid JC, Santaguida LP. Standardization of adverse event terminology and reporting in orthopaedic physical therapy: application to the cervical spine. J Orthop Sports Phys Ther 2010:40:455-63.

39. Poreisz C, Boros K, Antal A, et al. Safety aspects of transcranial direct current stimulation concerning healthy subjects and patients. Brain Res Bull 2007;72:208-14.

40. Song CY, Lin SF, Huang CY, et al. Validation of the brief pain inventory in patients with low back pain. Spine 2016:41:E937-E942.

41. Ger LP, Ho ST, Sun WZ, et al. Validation of the brief pain inventory in a Taiwanese population. J Pain Symptom Manage 1999;18:316-22.

42. Chapman JR, Norvell DC, Hermsmeyer JT, et al. Evaluating common outcomes for measuring treatment success for chronic low back pain. Spine 2011;36(21 Suppl):S54-S68

43. Jordan K, Dunn KM, Lewis M, et al. A minimal clinically important difference was derived for the Roland-Morris Disability Questionnaire for low back pain. J Clin Epidemiol 2006;59:45-52.

44. Hassett LM, Moseley AM, Tate RL, et al. Efficacy of a fitness centrebased exercise programme compared with a home-based exercise programme in traumatic brain injury: a randomized controlled trial. $J$ Rehabil Med 2009;41:247-55.

45. Vuilleumier PH, Biurrun Manresa JA, Ghamri Y, et al. Reliability of quantitative sensory tests in a low back Pain Population. Reg Anesth Pain Med 2015:40:665-73.

46. Walton DM, Macdermid JC, Nielson W, et al. Reliability, standard error, and minimum detectable change of clinical pressure pain threshold testing in people with and without acute neck pain. $J$ Orthop Sports Phys Ther 2011;41:644-50.

47. Moloney NA, Hall TM, Doody CM. Reliability of thermal quantitative sensory testing: a systematic review. J Rehabil Res Dev 2012;49:191-207.

48. Klyne DM, Schmid AB, Moseley GL, et al. Effect of types and anatomic arrangement of painful stimuli on conditioned pain modulation. J Pain 2015;16:176-85.

49. Rabey M, Slater H, O'Sullivan P, et al. Somatosensory nociceptive characteristics differentiate subgroups in people with chronic low back pain: a cluster analysis. Pain 2015;156:1874-84

50. Scholz J, Mannion RJ, Hord DE, et al. A novel tool for the assessment of pain: validation in low back pain. PLoS Med 2009;6:e1000047.

51. Owens MA, Bulls HW, Trost Z, et al. An examination of pain catastrophizing and endogenous pain modulatory processes in adults with chronic low back pain. Pain Med 2016:17:1452-64.

52. Geber C, Klein T, Azad S, et al. Test-retest and interobserver reliability of quantitative sensory testing according to the protocol of the German Research Network on Neuropathic pain (DFNS): a multicentre study. Pain 2011;152:548-56.

53. Van Breukelen GJ. ANCOVA versus change from baseline: more power in randomized studies, more Bias in nonrandomized studies [corrected]. J Clin Epidemiol 2006;59:920-5.

54. Abbott $\mathrm{JH}$. The distinction between randomized clinical trials (RCTs) and preliminary feasibility and pilot studies: what they are and are not. J Orthop Sports Phys Ther 2014;44:555-8.

55. Ribeiro DC, Sole G, Abbott JH, et al. The effectiveness of a lumbopelvic monitor and feedback device to change postural behavior: a feasibility randomized controlled trial. J Orthop Sports Phys Ther 2014;44:702-11. 